\title{
Efectos del Estrés en el Rendimiento Académico de los Estudiantes de la Licenciatura de Psicología
}

\author{
Effects of Stress on the Academic Performance of Students of the Bachelor \\ of Psychology
}

\section{Mónica Gabriela Barrón Miranda ${ }^{1}$, Lizeth Armenta Zazueta ${ }^{2}$}

${ }^{1}$ Ramón Corona s/n y Aguascalientes Col. Itson

Cel. 6421358574

barron_monica@hotmail.com

Instituto Tecnológico de Sonora

ORCID: https://orcid.org/0000-0003-2600-4934

${ }^{2}$ Mariano Escobedo 1208 Col. Itson

Cel. 6421179825

Lizeth.armenta@itson.edu.mx

Instituto Tecnológico de Sonora

ORCID: https://orcid.org/0000-0002-9073-2461

DOI https://doi.org/10.46589/rdiasf.vi36.388

Recibido 2 de junio 2021.

Aceptado 20 de septiembre 2021

Publicado 28 de octubre 2021

\section{Resumen}

Esta investigación se llevó a cabo debido a la gran interrogante que existe de cómo es que afecta el estrés en el rendimiento académico de los estudiantes. Teniendo como objetivo determinar los efectos del estrés en el rendimiento de los estudiantes de la licenciatura en psicología a través de la aplicación de un instrumento que determinará la asociación entre dichas variables. Con este instrumento se utilizó el método cuantitativo, el cual permitió identificar las variables con mayor incidencia que afecta el rendimiento académico de los 
alumnos. Esto mediante la recolección de los datos a través de una encuesta en línea. Fueron 33 los participantes de la investigación, de los cuales se obtuvo como resultado que el $69.7 \%$ de los alumnos presenta estrés medio, $18.2 \%$ estrés alto y el 12.1 estrés bajo. Se llegó a la conclusión que son muchas las causas y los efectos que generan estrés y esto influye en el rendimiento académico de los estudiantes. Algunas de ellas son la carga excesiva de tareas y trabajos con altos niveles de exigencia, el tiempo limitado para hacer dichas actividades, trastorno de sueño, problemas de concentración para realizar cualquier actividad. Estos pueden ser generados por diversos factores, dependiendo de las circunstancias de cada estudiante, el entorno social como las relaciones familiares.

Palabras clave: rendimiento académico, estrés, estudiantes.

\begin{abstract}
This research was carried out due to the great question that exists of how stress affects the academic performance of students. Aiming to determine the effects of stress on the performance of undergraduate students in psychology through the application of an instrument that will determine the association between these variables. With this instrument the quantitative method was used, which allowed to identify the variables with the highest incidence that affect the academic performance of the students. This by collecting the data through an online survey. There were 33 research participants, of which it was obtained as a result that $69.7 \%$ of the students present medium stress, $18.2 \%$ high stress and 12.1 low stress. It was concluded that there are many causes and effects that generate stress and this influences the academic performance of students. Some of them are the excessive load of tasks and work with high levels of demand, the limited time to do these activities, sleep disorder, concentration problems to carry out any activity. These can be generated by various factors, depending on the circumstances of each student, the social environment and family relationships.
\end{abstract}

Keywords: academic performance, stress, students.

\title{
Introducción
}

ISSN: 2007-8870

Universidad de Sonora
Los contenidos de este artículo están bajo una licencia de Creative Commons Atribución No Comercial - Sin Obra Deriva da 4.0 Internacional 


\section{Objetivo:}

Determinar los efectos del estrés en el rendimiento académico de los estudiantes de la Licenciatura de Psicología a través de la aplicación de un instrumento que determinará la asociación entre dichas variables.

\section{Objetivo específico:}

Identificar las principales causas que generan estrés en los estudiantes.

\section{Preguntas de investigación:}

¿Cuáles son los efectos del estrés en el rendimiento académico de los estudiantes de la Licenciatura en Psicología?

¿Cuáles son las principales causas que generan estrés en los estudiantes?

\section{Limitaciones}

En toda investigación que se realice sobre cualquier tema, casi siempre se encontraran limitaciones las cuales surgen y limitan de alguna forma el proceso, la aplicación o los resultados de la investigación. En esta investigación para medir los efectos del estrés en el rendimiento académico de los estudiantes de la Licenciatura de Psicología. Solo se presentó una limitante la cual fue que los alumnos de dicha licenciatura al momento de que los investigadores fueron aplicar la encuesta, no se encontraban todos los alumnos debido a que estaban en tallares, aplicando encuesta, y los investigadores tuvieron que volver otro día para aplicar a los alumnos que faltaban.

\section{Justificación}

El tema de esta investigación "Efectos del estrés en el rendimiento académico de los estudiantes" tiene mucha relevancia en el contexto escolar debido que es un tema con mucha rama de investigación, porque puede ser aplicable para cualquier nivel educativo, carrera universitaria, y en cualquier lugar del mundo.

Este proyecto se llevó a cabo debido a la gran interrogante que existe, cómo afecta el estrés en el rendimiento de los estudiantes de la Licenciatura en Psicología y cuáles son sus principales causas que lo generan estrés. Con este estudio se beneficiará a la sociedad estudiantil de dicha Licenciatura, a la cual se les aportará los resultados que arroje el instrumento y con ello podrán entender mejor el porqué de las reacciones que se presentan ante distintas situaciones. Al finalizar considerando los resultados se propondrán estrategias 
de mejora, para disminuir lo mayor posible todo aquello que genere estrés y por ello afecte el desempeño como en el rendimiento escolar de los estudiantes.

Arnett (2000) citado en Pulido, y Cols. (2011) coincide con lo que menciona Tolentino (2009), señalando que el estrés académico alcanza sus estándares más altos en los estudios de nivel superior, ya que estos se caracterizan por altas cargas de trabajo, los cuales son solicitados con altos niveles de exigencia y calidad. Por esta razón, algunos de los estudiantes no soportan la presión y el estrés que la escuela, los maestros y compañeros les generan y prefieren abandonar la carrera, o aplazar más años de los que comúnmente son (Zeidner, 1992 citado por Kadapatti y Vijayalaxmi (2012).

\section{Sustento Teórico}

\section{Estrés en jóvenes estudiantes}

Hablar de estrés, es un tema que ha generado interés en la comunidad estudiantil, debido a que regularmente es donde el índice de elementos estresores va en aumento. Según Román y Hernández, (2011), el estrés surge en el siglo XVII, con el descubrimiento de que el estrés académico se genera principalmente en aspectos personales, como percepción, actitud hacia el trabajo, disposición de cumplir con las actividades académicas, y tiempo destinado a la realización de trabajos.

El estrés es un problema que afecta a todos por igual, no importa que sean niños, jóvenes, adultos, hombres, mujeres, tampoco importa que tengan una buena posición económica es más Caldera, Pulido y Martínez (2007) reitera que el estrés se encuentra en presente en todos los ambientes, medios y contextos, como en la escuela, familia, trabajo, en la sociedad. Ya que toda ser humano puede presentar en algún momento dada de su vida estrés, por diferentes factores que se le puedan presentar. Ya sea problemas en su vida personal como en su entorno.

Fernández y Luévano (2018), explican que el estrés tiene distintas variables, y diferentes etapas, dependiendo de la persona que los padece, ya que cada estudiante manifiesta su estrés de manera distinta. Además, el proceso de adaptación de cada persona varía según su experiencia, habilidades y aptitudes. Cada individuo tiende a interpretar y percibir diferente las situaciones. 
Actualmente, se considera el tema del estrés como causa de alteración en el desarrollo emocional, en la conducta, en las relaciones personales e interpersonales o simplemente en la salud física de los estudiantes (Pozos, Preciado, Plascencia, Acosta y Aguilera, 2015). Es importante que la mayoría o la totalidad del alumnado desarrolle al máximo su inteligencia emocional, debido a que las emociones son de las principales causas que genera estrés en la universidad y provoca un bajo rendimiento escolar.

\section{Estrés académico}

El estrés académico es provocado en el ámbito educativo. Cuando a un estudiante tiene actividades excesivas y que sobrepasan sus recursos, o sus capacidades, es cuando nace el estrés académico, afectando sus pensamientos, por lo que lleva a un aprendizaje limitado, actitudes negativas que le provocan al alumno disminución en sus esfuerzos y productividad. Esto se presenta en mayor medida cuando el docente lleva un alto nivel de exigencia con el alumno, y éste ya se encuentra saturado de trabajo. Si, además, se le agrega el factor motivacional nulo, puede generar frustración, bajo aprendizaje, baja calidad de sus trabajos académicos, y, por ende, bajo rendimiento escolar (García, Pérez y Natividad, 2011).

Para entender el estrés académico, principalmente, se tiene que considerar el entorno del estudiante, ya sean sus condiciones familiares, sociales, económicas, culturales e institucionales. Estas variables, son importantes, además de su actitud y manejo del temperamento. También influye su capacidad de resolución de conflictos, el contar con apoyo social y económico, pues es de los rasgos por el cual los alumnos dejan de estudiar o estudian bajo presión, solo por cumplir con una jornada laboral (Suárez y Díaz, 2014). En la universidad se considera que los jóvenes presentan mayor frecuencia estrés, debido a que se están preparando para su profesión que ejercerán en un futuro, y por lo tanto los docentes les exigen a sus estudiantes una buena elaboración de trabajos con responsabilidad. Tolentino (2009), explica que, durante el primer año de universidad, presentan mayor nivel de estrés, debido a la falta de control. No obstante, este puede superarse en el transcurso del tiempo en la universidad. De igual manera es considerado el último año de escolar, un causante de estrés, esto es debido a que los estudiantes están bajo presión académica a causas de los proyectos solicitados, prácticas profesionales, horas de 
servicio, proyecto de titulación y además exigencias de trabajos que llevan mayor preparación y dedicación (Encina y Ávila, 2015).

\section{Causas del estrés}

Existen muchos factores que pueden influir en una persona para generarle estrés entre alguno de ellos es la responsabilidad que tienen, ya sea en su vida personal, laboral o profesional, el clima de organización, la carga y ambiente de trabajo, no hacer lo que le gusta, entre otros. Barraza, (2007) menciona algunas de las causas físicas que genera el estrés en las personas son el incremento del pulso cardiaco, falta de sueño, roce de dientes, fatiga crónica, problemas de digestión, incremento en la tensión muscular de brazos y piernas.

Así mismo, indica que las causas psicológicas más comunes del estrés son la ansiedad, pérdida de confianza en ellos mismo, dificultad para tomar decisiones, caen en depresión, inquietud, perturbación, falta de concentración y preocupación. Y por último las causas conductuales son el aislamiento, deterioro del desempeño, desgano, consumo de bebidas alcohólicas, ausentismo, incremento o disminución del sueño, propensión a los accidentes entre otros. Todo ello es debido a que el estrés es un estresor que puede afectar no solo de una forma sino de muchas.

A continuación, se presentan algunos tipos de problemas que nos conducen al estrés: a) mala calidad de sueño: b) fatiga excesiva y presencia de "micro sueños" involuntarios; c) la memoria de corto plazo y de trabajo disminuye; d) disminución de la flexibilidad cognitiva; e) toma de decisiones más arriesgadas; f) el desempeño inicial de las tareas es bueno al inicio, pero se deteriora con las tareas de larga duración; g) alteración de la capacidad de juicio; h) disminución de la flexibilidad cognitiva; i) alteraciones del humor: irritabilidad, enojo, disminución de la auto vigilancia y autocritica.

\section{Enfermedades Asociadas al Estrés}

En las escuelas se debe tener en consideración la gran importancia del conocer lo que es el estrés y sus niveles en los estudiantes. El estrés académico es una de las principales problemáticas que se ven en los estudiantes ya que suelen tener más enfermedades, en esta etapa como una de las principales es la depresión, enfermedades cardiacas, falta de sueño, aquí según estudios los alumnos presentan faltas en clase, trabajos 
inconclusos, incluso algunos estudiantes se dan de baja en la carrera universitaria por esa gran problemática. Hoy en día la depresión es lo que se está presentando con más frecuencia en alumnos de universidad, ya que esto está enlazado con el estrés académico. Todo estudiante o persona cuando no se adaptan al trabajo excesivo que dejan los docentes es cuando surgen los problemas, y es porque el estudiante no se acostumbra fácilmente y no da el rendimiento que están exigiendo.

Esto que se está presentando hoy es día se tiene que tomar con mucha importancia debido a que es un gran peligro para los jóvenes encontrarse en esa situación, ya que cabe mencionar que todos llevan una vida social y no se sabe en qué momento emocional se encuentren ellos. Para ello sería de gran importancia que los docentes y directivos de las escuelas que buscaran alguna solución de poder ayudar a los estudiantes cuando se encuentren en esa situación o que se encontrara alguna solución para evitar este tipo de problemáticas que se están viendo hoy en día con más frecuencia en los estudiantes y profesionistas del futuro, (Pulido, Serrano, Valdés, Chávez, Hidalgo, Vera, 2011).

\section{Rendimiento Académico}

El rendimiento académico es el desenvolvimiento de un estudiante durante una jornada o tiempo establecido de trabajo y esto demuestra un resultado que indica cómo fue dicho rendimiento que llevó durante ese tiempo, el cual podrá ser bueno o malo. A continuación, se muestran algunos puntos importantes sobre el rendimiento académico:

1. Es una serie de factores que se presentan en la persona que está aprendiendo.

2. Es un resultado de como un estudiante está aprendiendo por las actividades asignadas de un profesor.

3. Son una serie de factores de orden social como por ejemplo emocional y cognitivo.

4. Es una calificación o nota obtenida durante el tiempo cursado en un semestre de los alumnos que hayan concluido ese periodo.

Según la variable demográfica en México son las mujeres las que tienen un mayor rendimiento académico, esto se dice ya que en comparación con los hombres las mujeres superan el rendimiento académico.

\section{Estresores en la Universidad}


Según se ha demostrado que el estrés aumenta cuando el estudiante progresa en sus estudios académicos, especialmente ocurre el estrés académico cuando al estudiante se le presenta en la universidad mucha carga excesiva de trabajos, pero de igual manera coinciden con la etapa que se le presente al estudiante o se enfrente con cambios en su vida. Un ejemplo es cuando el estudiante ingresa a una universidad externa al lugar donde él vive y es cuando entra el proceso de separación de su familia, la incorporación al mercado laboral y la adaptación del lugar a donde él se tenga que ir a adaptarse, es cuando en su mente se vuelve más vulnerable, porque ya no se encuentra en su zona de confort a lo que es su hogar o están en la creencia que se encuentra en un lugar muy extraño.

Entonces es cuando los estudiantes universitarios llegan a sufrir periodos de estrés. Así, dada la vulnerabilidad de los estudiantes universitarios a sufrir periodos de estrés sostenido (Pulido, Serrano, Valdés, Chávez, Hidalgo y Vera, 2011).

En la investigación de Pulido, Serrano, Valdés, Chávez, Hidalgo y Vera, (2011), dice que los estresores radican en algunos aspectos que afectan a la vida durante sus estudios se descubrieron mayores niveles de estrés en estudiantes cuando se presentan algunos factores los cuales son: a) viven fuera de la familia; b) los estilos de afrontamiento que se utilizan ante el estrés; c) uso de drogas legales o ilegales; d) exigencia de los docentes.

\section{Motivación}

La motivación es definida según como un componente psicológico que orienta, mantiene y determina la conducta de una persona. García y Basilotta (2017) indica que es como una voluntad de una persona al realizar algunas acciones o cambia en su comportamiento para alcanzar un objetivo por ejemplo cuando una persona al realizar algo sabe que obtendrá algún beneficio o algo en específico que él quiera es cuando entra la motivación entonces la persona hace lo necesario para alcázar ese objetivo.

También menciona en su artículo los tipos de motivaciones que según las distintas teorías y criterios existen (intrínseca, extrínseca y motivación en el aula), y hace énfasis en los tipos que surgen a raíz de los beneficios. A continuación, se mencionan los tres tipos de motivaciones.

\section{Motivación intrínseca}

ISSN: 2007-8870

Universidad de Sonora
Los contenidos de este articulo están bajo una licencia de Creative Commons Atribución No Comercial - Sin Obra Deriva da 4.0 Internacional 
Esta motivación dice que aquellas motivaciones que surgen de consecuencias internas por ejemplo que la referencias provienen de una consecuencia interna. Es decir, que cuando una persona siente y quiere obtener o decía tener algo hace lo posible por alcanzarlo (García, Basilotta, 2017).

Para Schunk (1997), citado por Alvarado (2015) la motivación intrínseca es aquella que se enfoca en el control y la competencia. Es cuando una persona se propone una meta o un objetivo y desarrolla retos para obtener esa recompensa o domina alguna situación que este dentro de su interés

\section{Motivación extrínseca}

La motivación extrínseca es lo adverso a la motivación intrínseca esta se produce por consecuencias externas. Están son las que se producen a través del entorno como son el pago de fin de mes, algún tipo de reconocimiento, algún bono, etc.

Para Alvarado (2015), la motivación extrínseca habla de objetivos que son como un castigo o recompensa. La mayoridad las personas se han relacionado con la motivación extrínseca y un ejemplo de esta motivación podría ser que cuando a una persona es castigada y a cambio de su buen comportamiento le otorgan alguna recompensa es cuando entran a ese tipo de motivación. Es decir que la motivación no sale de alguna persona en sí, es cuando las personas ayudan a algún individuo a mejorar en alguna situación.

\section{Motivación en el Aula}

Campo (2017) explica que los alumnos cuando están motivados prestan más atención a las clases y aprenden más, y en esa situación según el autor, los estudiantes se involucran en más actividades dentro del aula, como por ejemplo, suelen ser participativos en la clase son muy capaces de realizar cualquier tipo de actividad, se esfuerzan al máximo en materias que a ellos se les presenta alguna dificultad y esto es la realidad cuando un estudiante está motivado es cuando se adapta a cualquier tipo de trabajo. Alvares Rojo y otros (1999) citado por Murillo (2008 afirman que la motivación es la que lleva al estudiante al realizar buenos trabajos y a conservar esa actitud positiva durante el periodo de elaboración de cualquier actividad.

Por su parte, Murillo (2008) afirma que una buena motivación se da cuando surge un logro significante para el estudiante. Según Ormrod (2005), afirma que la motivación 
general, la motivación específica y el rendimiento académico están asociados significativamente. Como por ejemplo la motivación general es la que una persona se plantea como una idea de lo que podría hacer para logar alguna meta en específico y la motivación especifica es cuando se enfoca y trabaja algún envidio para cumplir ese objetivo.

Todo esto se relaciona con el rendimiento académico ya que para lograr la meta se debe de obtener una motivación general, por ejemplo, los estudiantes plantean su objetivo que es terminar un trabajo asignado para después usar la motivación especifica de cómo realizarlo y comenzar a trabajar para tener como fin que es la elaboración y terminación de un buen trabajo. Según Campo (2015), la motivación afecta a gran parte del aprendizaje y al rendimiento al menos en cuatro formas las cuales son:

1. El aumento en el nivel de energía y el nivel de actividad del individuo. Esto surge cuando una persona realice una actividad de forma intensa o activa y al mismo tiempo la realice con desgano.

2. Cuando a un estudiante lo dirigen a ciertas metas sin una buena motivación y esto hace que la persona realice esa actividad de forma mediocre ya que no la realiza con una buena actitud.

3. La motivación favorece al individuo en que inicia algo por su propia iniciativa, cuando la persona realiza una actividad con ánimos es cuando todo fluye de una manera correcta y cuando una persona realiza una actividad sin iniciativa, sin ánimos y sin motivación es cuando aparece el bajo rendimiento.

4. Afecta principalmente a las estrategias de aprendizaje, procesos cognitivos y a la autoestima del individuo al momento de realizar alguna tarea.

Escudero (1978) y Rivera (2014) entienden por motivación intrínseca al recurso con el que el estudiante ha de realizar una tarea dada, ya sea a factores vinculados de por sí a la tarea, ya sea a componentes de significación o afectivos. Berlyne (1960), Hunt (1961) y Bruner (1964) fueron los que realizaron el estudio de la motivación, tales guardan mucha relación con la cognitivista de la motivación y es la principal base en la que parten todas las teorías incluidas en el enfoque ya que la cognición es algo interno del sujeto. 
Por su parte, González, Valle, Núñez y González (2014) discuten que desde ya hace algunos años existe una relación de psicología del aprendizaje, que estudia de manera conjunta la cognición y la motivación, ya que se consideran como el aprendizaje como proceso cognitivo y motivacional a la vez. También resaltan que los estudiantes para que tengan buenos resultados académicos necesitan aportar dos grandes puntos importantes tales como son la voluntad y la habilidad con estas dos grandes virtudes del individuo al momento de realizar algún trabajo o actividad tendrán como fin buenos resultados académicos ya que la voluntad viene acompañada de la motivación que posee una persona y la habilidad es como teniendo esa motivación la persona tiene buenos resultados en sus actividades.

En cualquier tarea, los alumnos motivados de forma intrínseca suelen: a) hacer la tarea por iniciativa propia, sin que haya que empujarlos a ella; b) implicarse cognitivamente en la tarea; c) abordar aspectos más difíciles de la tarea, d) aprender la información de forma significativa y no de memoria; d) ser creativos durante la ejecución; e) persistir a pesar del fracaso; f) buscar oportunidades adicionales para seguir con la tarea y g) tener un alto rendimiento académico (Rivera, 2015).

\section{Asociación del Estrés al Rendimiento Académico}

El estrés también suele estar presente en situaciones personales y en lo familiar como son problemas en casa, con amigos incluso con algún trabajo y es cuando se presentan más problemas como alejamiento con las personas que quieres por la situación en la que te encuentras y hasta perder cosas que realmente quieres gracias a esta. Según Basnet, Jaiswal, Adhikari y Shyangwa (2013), los principales problemas que causan el estrés son que te alejas de las personas que quieres por qué piensas que todo te estorba y te excluyes de la sociedad como amigos, la familia y los seres que más quieres. Esto pasa ya que cuando una persona se encuentra en esa situación lo único que busca es paz y tranquilidad se encierra en su entorno y no busca una solución al problema para volver a estar bien y la única solución que a él se le presenta es estar solo.

Algunos estudios relacionan las alteraciones emocionales de las personas con el estrés académico y es cuando surgen diferentes emociones como son: a) enojo; b) miedo; c) ansiedad; d) nerviosismo; e) depresión. 
El estrés se pude considerar como un factor educativo y laboral; este se define como una reacción fisiológica, emocional, cognitiva y conductual ante una serie de impulsos y cuestiones de carácter académicos (Berrío, Mazo 2011). Según Román y Hernández (2011) la función del estrés en las personas es la de alterar sus niveles cognitivos, fisiológicos y conductuales, provocando en ellas una perspectiva negativa y por ende, un rendimiento bajo en el ámbito educativo, debido a una presión que se provoca en la misma generándose una barrera imaginaria la cual le impide avanzar y realizar sus actividades, disminuyendo sus calificaciones por su causa y en casos extremos, provoca en el alumno un desinterés o desmotivación para asistir a clases por lo que el estudiante no se presenta en clases.

Sánchez, Oviedo y Martínez, (2011) el concepto del síndrome del estrés es un signo o síntoma englobado al estado morboso, y este se encuentra accesible a la examinación psicopatológica. De acuerdo con diversos factores en el estrés, los síndromes se clasifican en tres tipos, tales como:

1. Inespecíficos: que son aquellos más o menos crónicos de sobrecargas, provocando en la persona una reducción en la capacidad defensiva y el aumento en la fragilidad al organismo.

2. Semi-específicos: en ellos, se relacionan los aspectos externos del estrés a otros factores contagiosos como: psicosis reactivas, reacciones alérgicas, trastornos de ansiedad, entre otros.

3. Específicos: estos son factores externos del síndrome del estrés determinados en el origen del proceso morboso de la persona. Los síndromes más comunes en estos grupos son los siguientes: el estrés postraumático, trastorno agudo de estrés y los trastornos adaptados.

Los estudiantes aprenden de distintas maneras, por lo tanto, es importante reconocer la finalidad de que los maestros establezcan estrategias que posibiliten el proceso de aprendizaje, y estos adquieran conocimientos, mejorando entonces su rendimiento (Sánchez, Oviedo y Martínez, 2011). Se dice que el rendimiento académico no es producto de una aptitud, sino que se trata de un efecto sintético de una serie de factores que influyen en él, desde la persona que aprende.

Desde una percepción educativa, se reconoce al rendimiento académico como una calificación o resultado medio obtenido durante el periodo escolar que haya curado el 
alumno, cuyas variables que implican dentro del mismo se derivan en: demográficas (sexo, edad, estado civil, experiencia laboral), variables académicas (estudios cursados, curso, opción de la carrera a cursar o que se cursa, rendimiento anticipado) y variables socio familiares (grado de estudio de los padres de familia, la satisfacción laboral de los mismos, su residencia familiar, lugares de estudio) Sánchez, Oviedo, Martínez (2011).

\section{Influencia de las emociones en el rendimiento académico según Pulido y Herrera (2017):}

Actualmente aparecen problemas dentro del ámbito educativo, los cuales los profesores no pueden contestar de forma efectiva, ya que son de carácter emocional y no se pueden determinar de una forma exacta los factores. Cualquier intervención dentro del ámbito escolar debe fundamentarse en la predictibilidad y uniformidad, no obstante, los estados emocionales no pueden borrarse; por lo que se propone alcanzar una mayor calidad con eficiencia en el proceso de enseñanza y aprendizaje de los alumnos, añadir las emociones en cualquier dimensión que interviene dentro de dicho proceso. El rendimiento académico de los estudiantes es, por su dificultad y relevancia, uno de los problemas con mayor discusión dentro de la investigación educativa, a la cual se le ha dedicado una atención específica dentro de las últimas décadas. Es una problemática que preocupa a estudiantes, padres de familia, docentes y autoridades; no sólo en México, sino en muchos países latinoamericanos (Lamas, 2015).

El rendimiento académico, es reconocido por su capacidad clasificatoria y el vínculo que tiene hacia la examinación de estudiantes, las notas y los promedios académicos identifican a este con objetividad. No obstante, no es algo legal, existen factores intrínsecos y sociales que intervienen en el mismo (Erazo, 2012). Sin lugar a dudas, existen indicadores que dan cuenta a los aprendizajes de los estudiantes en contextos educativos, su rendimiento correspondiente a la naturaleza multidimensional que caracteriza al mismo sigue en el intento de determinar sus variables influyentes que afectan y hacen modificar el rendimiento.

En la actualidad, parece ser asumido por integrantes de toda la comunidad educativa, la educación debe estar enfocada en generar competencias y habilidades que cada alumno debe poseer para aplicar en su vida cotidiana, para ello, se cuenta con una 
reforma educativa. Así mismo, el interés incrementado por conocer las competencias de los estudiantes que son como indicadores de sus logros escolares, y a su vez, sirva como guía en el movimiento de los estudios educativos (González, Nieblas, Díaz, López 2012).

Es un hecho tomar en cuenta que el rendimiento académico se determina por una serie de factores o variables que son procedentes de diferentes contextos como: personales, familiares, educativos y sociales. Es una medida de cada una de las habilidades de los estudiantes, que expresan lo que han adquirido a lo largo del proceso formativo del curso; también conlleva las competencias de los alumnos para responder a diferentes estímulos de carácter escolares; es un pilar de suma importancia para aquellos profesionales que se dediquen a la docencia, es muy necesario para el papel que desarrollarán los egresados de forma profesional, según Molina (2015) y Vázquez (2005).

\section{Método}

\section{Diseño de investigación}

Para efectos de este estudio, se utilizó el método cuantitativo, a través de la recolección de datos con un cuestionario llamado estrés y rendimiento académico en estudiantes de Psicología (Barraza, 2010). El procesamiento de los datos fue con el paquete estadístico Statistical Package for the Social Sciences (SPSS) versión 23. El diseño fue no experimental, descriptivo, retrospectivo y transversal (Hernández, Fernández y Baptista, 2014).

Este diseño permitió recolectar los datos dentro del ambiente universitario a través de una encuesta en línea, con que se pudieron describir, medir y cuantificar las variables estrés y el avance del rendimiento académico.

\section{Población y muestra}

Actualmente la carrera de Psicología, según datos del Sistema Integral de Trayectorias (SITE, 2019) cuenta con 205 alumnos inscritos, y 33 estuvieron dispuestos a participar en el estudio, siendo de segundo, cuarto, sexto semestre, tal como se muestra en la tabla 1 .

Tabla 1

Participación de alumnos por semestre. 


\begin{tabular}{llll}
\hline Semestre & Cantidad & Porcentaje & Porcentaje válido \\
\hline Segundo & 14 & 42.4 & 45.2 \\
Cuarto & 4 & 12.1 & 12.9 \\
Sexto & 13 & 39.4 & 41.9 \\
No respondieron & 2 & 28.4 & \\
Total & 33 & 100.0 & 100.0
\end{tabular}

La muestra se obtuvo a través de una invitación por escrito, donde cada uno de los participantes firmó una carta de consentimiento informado en la cual aceptan ser parte del estudio. Como criterio de inclusión se consideraron a los estudiantes matriculados en la carrera de Psicología, en cualquier semestre, segundo, cuarto, sexto.

\section{Instrumento}

Para la recolección de datos, se tomó como referencia un instrumento llamado estrés y rendimiento académico en estudiantes de Psicología, (Barraza, 2010), con adecuaciones leves de lenguaje para fines de este estudio (ver anexo 1). De esta manera, pudieron obtenerse datos relacionados al estrés académico y el rendimiento escolar de cada uno de los participantes.

El instrumento fue obtenido, estandarizado y adaptado del inventario SISCO (Barraza, 2010), donde se miden tres niveles de estrés que se describen en la tabla 2, y que proporcionan un aproximado del grado de estrés en el que cada estudiante se sitúa. El instrumento cuenta con 26 ítems, donde 23 de ellos cuenta con el intervalo de respuesta: nunca, 1 punto; rara vez, 2 puntos; algunas veces, 3 puntos; casi siempre, 4 puntos y siempre, 5 puntos, y las puntuaciones van acordes a la tabla 2. Además, una pregunta dicotómica para determinar el género del participante, una pregunta de opción múltiple para conocer el semestre en el que está inscrito y otra de opción múltiple para conocer su promedio actual.

Tabla 2.

Valores y niveles de estrés.

\begin{tabular}{ll}
\hline Nivel de estrés & Puntuación \\
\hline Estrés bajo & $10-39$ \\
\hline
\end{tabular}




\begin{tabular}{ll}
\hline Estrés medio & $40-79$ \\
Estrés alto & $80-115$
\end{tabular}

Para poder definir el rendimiento académico, se tomó en consideración el criterio de la universidad, que es de cero a 100, donde el mínimo aprobatorio es 70, acorde al Plan de Desarrollo Institucional (PDI, 2015). De igual manera, está categorizado según a Gómez y Sánchez (2013), con las siguientes valoraciones: 70-80 bajo, 80-90 regular, y de 90-100 bueno. Con el instrumento aplicado se evalúan dos variables: el estrés y el rendimiento académico. Para la variable estrés se consideran la dimensión fisiológica, con seis ítems, dimensión comportamental, con cuatro ítems, y dimensión psicológica, con cinco ítems, haciendo una clasificación de estrés bajo, medio y alto (ver tabla 1), con una escala interválica y está constituido de 15 preguntas descritas anteriormente (ver anexo 2). Para la variable de rendimiento académico, las dimensiones que se consideran son bajo, regular y bueno, con una escala ordinal y un promedio ponderado.

En relación con la validez y confiabilidad, fue valorado con el coeficiente alfa de Cronbach, con una puntuación de .911 (ver tabla 3), lo cual, acorde a Hernández, Fernández y Baptista (2014), es un nivel aceptable y confiable para aplicarse.

Tabla 3.

Confiabilidad del instrumento aplicado.

\begin{tabular}{ll}
\hline Alfa de Cronbach & N de elementos \\
\hline .911 & 25 \\
\hline
\end{tabular}

\section{Procedimiento}

1. Se hizo la solicitud por escrito al Responsable de Programa Educativo para realizar el estudio con los alumnos matriculados en la carrera de Psicología.

2. Se digitalizó el instrumento que se les aplicó a los participantes.

3. Se les informó de manera presencial a los estudiantes matriculados en la licenciatura sobre este estudio y la importancia de su participación, así como el proceso a seguir.

4. Se envió a cada uno de los estudiantes, al correo que se tiene en el departamento de registro escolar. 


\section{Resultados}

En este capítulo se describen los resultados obtenidos de cada uno de los ítems del instrumento "Estrés y rendimiento académico en estudiantes de educación superior" el cual fue aplicado a un total de 33 alumnos, de segundo, cuarto y sexto semestre, inscritos en la carrera de Psicología, en el Instituto Tecnológico de Sonora.

Primeramente, se les solicitaba a los alumnos que marcaran con una $\mathrm{X}$ el sexo que los identificara, ya sea masculino o femenino. El resultado obtenido fue el $36.4 \%$ de sexo masculino y el otro $63.6 \%$ de sexo femenino (ver tabla 4 ).

Tabla 4.

Sexo

\begin{tabular}{llll}
\hline Sexo & Frecuencia & Porcentaje & Porcentaje válido \\
\hline Masculino & 12 & 36.4 & 36.4 \\
Femenino & 21 & 63.6 & 63.6 \\
Total & 33 & 100.0 & 100.0 \\
\hline
\end{tabular}

$\mathrm{Al}$ igual en el instrumento se les solicitaba a los alumnos que escribieran el semestre que actualmente están cursando. Los resultados obtenidos fueron los siguientes: el $42.4 \%$ del total de los alumnos está cursando segundo semestre, solo el $12.1 \%$ son de cuarto y el $39.4 \%$ son de sexto semestre (ver tabla 5).

\section{Tabla 5 .}

Semestre

\begin{tabular}{llll}
\hline Semestre & Frecuencia & Porcentaje & Porcentaje válido \\
\hline Segundo semestre & 14 & 42.4 & 45.2 \\
Cuarto semestre & 4 & 12.1 & 12.9 \\
Sexto semestre & 13 & 39.4 & 41.9 \\
Total & 31 & 93.9 & 100.0 \\
Perdidos & 2 & 6.1 & \\
Total & 33 & 100.0 &
\end{tabular}

Con el primer ítem se pretende conocer la frecuencia en que los alumnos se sienten inquietos frente a la competencia de los compañeros de grupo, se obtuvo como resultado, el $18.2 \%$ de los alumnos respondió que nunca, el 33.3\% rara vez, el 39.4\% algunas veces, el $6.1 \%$ casi siempre y solamente el $3 \%$ contestó que nunca (ver tabla 6). 


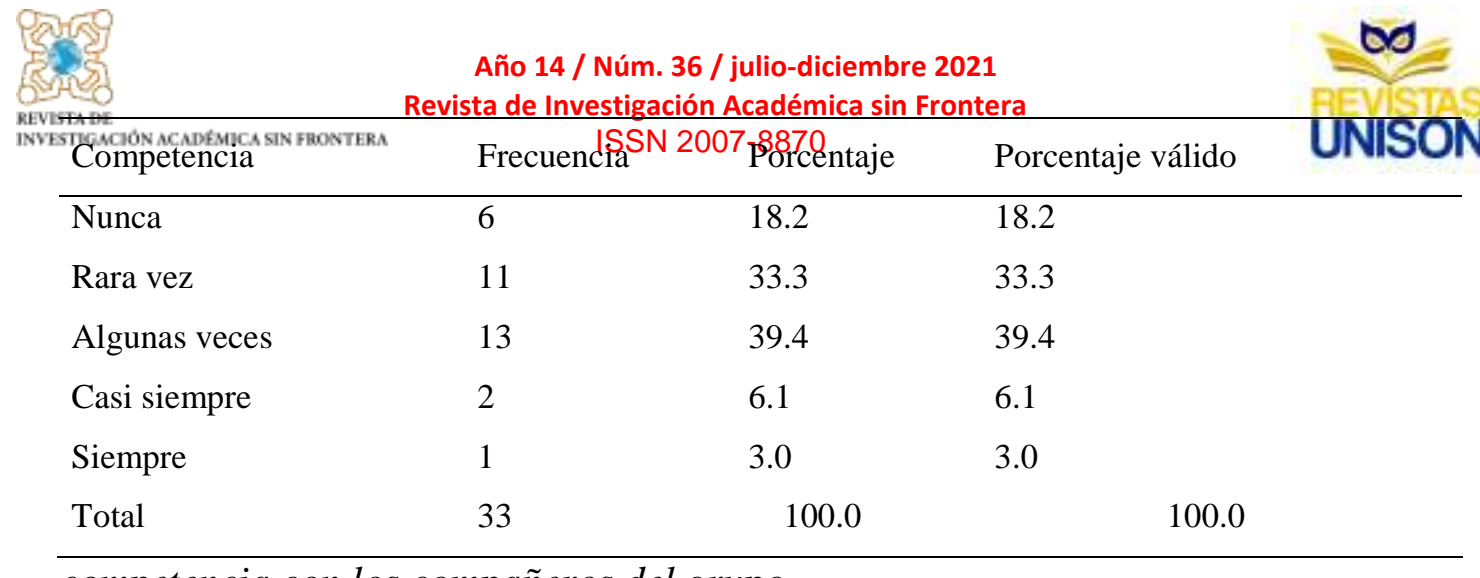

competencia con los compañeros del grupo

El segundo ítem pretende conocer la frecuencia en la que los alumnos se siente inquietos cuando tienen sobrecarga de tareas y trabajos, solamente el 3\% respondió que nunca, el $18.2 \%$ rara vez, el $21.2 \%$ algunas veces, el $36.4 \%$ casi siempre y el $21.2 \%$ respondió que siempre (ver tabla 7).

Tabla 7

La sobrecarga de tareas y trabajo

\begin{tabular}{llll}
\hline Sobrecarga de tareas & Frecuencia & Porcentaje & Porcentaje válido \\
\hline Nunca & 1 & 3.0 & 3.0 \\
Rara vez & 6 & 18.2 & 18.2 \\
Algunas veces & 7 & 21.2 & 21.2 \\
Casi siempre & 12 & 36.4 & 36.4 \\
Siempre & 7 & 21.2 & 21.2 \\
Total & 33 & 100.0 & 100.0 \\
\hline
\end{tabular}

El tercer ítem dará a conocer la frecuencia con la que los jóvenes se sienten inquietos con el carácter del docente, el 24.2\% contestó que rara vez, el 36.4\% algunas veces, el $33.3 \%$ casi siempre y únicamente el $6.1 \%$ contestó que siempre (ver tabla 8). Tabla 8.

El carácter del docente

\begin{tabular}{llll}
\hline El carácter del docente & Frecuencia & Porcentaje & Porcentaje válido \\
\hline Rara vez & 8 & 24.2 & 24.2 \\
Algunas veces & 12 & 36.4 & 36.4 \\
\hline
\end{tabular}




$\begin{array}{llll}\text { Casi siempre } & 11 & 33.3 & 33.3 \\ \text { Siempre } & 2 & 6.1 & 6.1 \\ \text { Total } & 33 & 100.0 & 100.0\end{array}$

Con el cuarto ítem se determina la frecuencia con la que los estudiantes se sienten inquietos en las evaluaciones de los docentes (exámenes, trabajos de investigación). Solo el $3 \%$ respondió que nunca, el $15.2 \%$ rara vez, $36.4 \%$ algunas veces, $27.3 \%$ casi siempre y el $18.2 \%$ restante respondió que siempre (ver tabla 9).

Tabla 9

Las evaluaciones de los docentes (exámenes, trabajos de investigación)

\begin{tabular}{llll}
\hline Evaluaciones de los docentes & Frecuencia & Porcentaje & Porcentaje válido \\
\hline Nunca & 1 & 3.0 & 3.0 \\
Rara vez & 5 & 15.2 & 15.2 \\
Algunas veces & 12 & 36.4 & 36.4 \\
Casi siempre & 9 & 27.3 & 27.3 \\
Siempre & 6 & 18.2 & 18.2 \\
Total & 33 & 100.0 & 100.0 \\
\hline
\end{tabular}

En el quinto ítem, se les cuestionaba a los alumnos la frecuencia con la que se inquietan cuando el tipo de trabajo que les pueden los docentes (fichas de trabajo, informes, etc.). Como resultado se obtuvo lo siguiente: solo el 3\% respondió que nunca, $18.2 \%$ rara vez, el 39.4\% algunas veces, el 15.2\% casi siempre y el 24.2\% restante respondió que siempre (ver tabla 10).

Tabla 10

El tipo de trabajo que te piden los docentes (fichas de trabajo, informes, etc.)

\begin{tabular}{lccc}
\hline Trabajo que piden los docentes & Frecuencia & Porcentaje & Porcentaje válido \\
\hline Nunca & 1 & 3.0 & 3.0 \\
Rara vez & 6 & 18.2 & 18.2 \\
Algunas veces & 13 & 39.4 & 39.4 \\
Casi siempre & 5 & 15.2 & 15.2 \\
Siempre & 8 & 24.2 & 24.2 \\
Total & 33 & 100.0 & 100.0
\end{tabular}

El sexto ítem tiene la finalidad de conocer la frecuencia en la que los estudiantes les inquieta no entender los temas que se abordan en clases, en ello los resultados obtenidos 
fueron los siguientes: el $18.2 \%$ de los alumnos contestaron que nunca al igual que rara vez, el $21.2 \%$ algunas veces, el $30.3 \%$ casi siempre y únicamente el $12.1 \%$ respondió que siempre (ver tabla 11).

Tabla 11

No entender los temas que se abordan en clase

\begin{tabular}{llll}
\hline No entender los temas & Frecuencia & Porcentaje & Porcentaje válido \\
\hline Nunca & 6 & 18.2 & 18.2 \\
Rara vez & 6 & 18.2 & 18.2 \\
Algunas veces & 7 & 21.2 & 21.2 \\
Casi siempre & 10 & 30.3 & 30.3 \\
Siempre & 4 & 12.1 & 12.1 \\
Total & 33 & 100.0 & 100.0 \\
\hline
\end{tabular}

De igual manera en este ítem se pretendía conocer la frecuencia con la que los alumnos se inquietan al participar en clase (responder a preguntas, exposiciones, etc.). Se obtuvo como resultado que el $18.2 \%$ nunca, $24.2 \%$ rara vez, $18.2 \%$ algunas veces, $27.3 \%$ casi siempre y el $12.1 \%$ restante contestó que siempre (ver tabla 12).

Tabla 12

Participación en clase (responder a preguntas, exposiciones, etc.)

\begin{tabular}{llll}
\hline Participación en clase & Frecuencia & Porcentaje & Porcentaje válido \\
\hline Nunca & 6 & 18.2 & 18.2 \\
Rara vez & 8 & 24.2 & 24.2 \\
Algunas veces & 6 & 18.2 & 18.2 \\
Casi siempre & 9 & 27.3 & 27.3 \\
Siempre & 4 & 12.1 & 12.1 \\
Total & 33 & 100.0 & 100.0
\end{tabular}

El siguiente ítem cuestionaba la frecuencia con la que los estudiantes se inquietan al tiempo limitado para hacer el trabajo, en el cual se obtuvo como resultado lo siguiente: el $3 \%$ de la totalidad del alumnado contesto que nunca, el $24.2 \%$ rara vez, el $21.2 \%$ algunas veces, el $36.2 \%$ casi siempre y el $15.2 \%$ siempre (ver tabla 13 ).

Tabla 13

Tiempo limitado para hacer el trabajo 


\begin{tabular}{llll}
\hline Tiempo limitado & Frecuencia & Porcentaje & Porcentaje válido \\
\hline Nunca & 1 & 3.0 & 3.0 \\
Rara vez & 8 & 24.2 & 24.2 \\
Algunas veces & 7 & 21.2 & 21.2 \\
Casi siempre & 12 & 36.4 & 36.4 \\
Siempre & 5 & 15.2 & 15.2 \\
Total & 33 & 100.0 & 100.0
\end{tabular}

Los siguientes ítems se dividen en tres apartados para conocer la frecuencia con la tienen reacciones físicas, psicológicas y comportamentales los jóvenes universitarios al estudiar la carrera de psicología en las labores académicas en su trayectoria escolar.

Primeramente, se quiere conocer las reacciones físicas y para ello se aplicaron seis ítems, con el primer ítem se procura conocer la frecuencia con la que los estudiantes presentan trastornos en el sueño (insomnio o pesadillas), los resultados generados son los siguientes: el 24.2\% afirma que nunca, al igual que rara vez y algunas veces, el $21.2 \%$ casi siempre y únicamente el $6.1 \%$ siempre presenta trastornos en el sueño (ver tabla 14). Tabla 14

Trastornos en el sueño (insomnio y pesadillas)

\begin{tabular}{llll}
\hline Trastornos en el sueño & Frecuencia & Porcentaje & Porcentaje válido \\
\hline Nunca & 8 & 24.2 & 24.2 \\
Rara vez & 8 & 24.2 & 24.2 \\
Algunas veces & 8 & 24.2 & 24.2 \\
Casi siempre & 7 & 21.2 & 21.2 \\
Siempre & 2 & 6.1 & 6.1 \\
Total & 33 & 100.0 & 100.0 \\
\hline
\end{tabular}

En el siguiente ítem se quiere conocer la frecuencia con la que los universitarios presentan fatiga crónica (cansancio permanente) en las actividades escolares, el $30.3 \%$ contestó que nunca al igual que rara vez, el 15.2\% algunas veces, el $21.2 \%$ casi siempre y solo el 3\% respondió que siempre (ver tabla 15).

Tabla 15

Fatiga crónica (Cansancio permanente)

\begin{tabular}{llll}
\hline Fatiga crónica & Frecuencia & Porcentaje & Porcentaje válido \\
\hline Nunca & 10 & 30.3 & 30.3
\end{tabular}


Rara vez

Algunas veces

Casi siempre

Siempre

Total
10

5

7

1

33
30.3

15.2

21.2

3.0

100.0
30.3

15.2

21.2

3.0

100.0

Este ítem se aplicó para determinar la frecuencia que tienen los estudiantes dolores de cabeza o migraña, el $21.2 \%$ respondió que nunca, el $27.3 \%$ rara vez, al igual que casi siempre, el $15.2 \%$ algunas veces y solo el $9.1 \%$ dijo que siempre (ver tabla 16).

Tabla 16

Dolores de cabeza o migraña

\begin{tabular}{llll}
\hline Dolores & Frecuencia & Porcentaje & Porcentaje válido \\
\hline Nunca & 7 & 21.2 & 21.2 \\
Rara vez & 9 & 27.3 & 27.3 \\
Algunas veces & 5 & 15.2 & 15.2 \\
Casi siempre & 9 & 27.3 & 27.3 \\
Siempre & 3 & 9.1 & 9.1 \\
Total & 33 & 100.0 & 100.0 \\
\hline
\end{tabular}

Este ítem tiene la finalidad de conocer la frecuencia con la que los universitarios presentan problemas de digestión, dolor abdominal o diarrea cuando tienen actividades escolares, el 39.4\% respondió que nunca, el 33.3\% rara vez, el 15.2\% algunas veces, el 9.1\% casi siempre y solamente el $3 \%$ contestó que siempre (ver tabla 17).

Tabla 17

Problemas de digestión, dolor abdominal o diarrea

\begin{tabular}{llll}
\hline Problemas de digestión & Frecuencia & Porcentaje & Porcentaje válido \\
\hline Nunca & 13 & 39.4 & 39.4 \\
Rara vez & 11 & 33.3 & 33.3 \\
Algunas veces & 5 & 15.2 & 15.2 \\
Casi siempre & 3 & 9.1 & 9.1 \\
Siempre & 1 & 3.0 & 3.0 \\
Total & 33 & 100.0 & 100.0 \\
\hline
\end{tabular}

Con este ítem se pretendía conocer la frecuencia con la que los jóvenes universitarios se muerden las uñas cuando se les presentas labores académicas, el $48.5 \%$ 
contestó que nunca, el $12.1 \%$ rara vez, el 27.3\% algunas veces al igual que casi siempre y el $9.1 \%$ siempre (ver tabla 18 ).

Tabla 18

Morderse las uñas

\begin{tabular}{llll}
\hline Morderse las uñas & Frecuencia & Porcentaje & Porcentaje válido \\
\hline Nunca & 16 & 48.5 & 48.5 \\
Rara vez & 4 & 12.1 & 12.1 \\
Algunas veces & 4 & 12.1 & 12.1 \\
Casi siempre & 6 & 18.2 & 18.2 \\
Siempre & 3 & 9.1 & 9.1 \\
Total & 33 & 100.0 & 100.0 \\
\hline
\end{tabular}

Este ítem tiene la finalidad de conocer la frecuencia con la que los estudiantes presentan somnolencia o mayor necesidad de dormir cuando tienen actividades académicas, el $21.2 \%$ indico que nunca, el $15.2 \%$ rara vez, $27.3 \%$ algunas veces al igual que casi siempre y el $9.1 \%$ siempre (ver tabla 19).

Tabla 19

Somnolencia o mayor necesidad de dormir

\begin{tabular}{llll}
\hline Somnolencia & Frecuencia & Porcentaje & Porcentaje válido \\
\hline Nunca & 7 & 21.2 & 21.2 \\
Rara vez & 5 & 15.2 & 15.2 \\
Algunas veces & 9 & 27.3 & 27.3 \\
Casi siempre & 9 & 27.3 & 27.3 \\
Siempre & 3 & 9.1 & 9.1 \\
Total & 33 & 100.0 & 100.0 \\
\hline
\end{tabular}

De igual forma se quiere conocer las reacciones psicológicas y para ello se aplicaron cinco ítems. Con el primer ítem de este apartado se desea conocer la frecuencia con las que los alumnos presentan inquietud (incapacidad de relajarse y estar tranquilos), en las labores escolares. Los resultados arrojados son los siguientes: el 18.2\% de los alumnos respondió que nunca, el $27.3 \%$ rara vez, el $27.3 \%$ algunas veces al igual que casi siempre, el $21.2 \%$ algunas veces y solo el $6.1 \%$ respondió siempre (ver tabla 20).

Tabla 20 
Inquietud (incapacidad de relajarse y estar tranquilos)

\begin{tabular}{llll}
\hline Inquietud & Frecuencia & Porcentaje & Porcentaje válido \\
\hline Nunca & 6 & 18.2 & 18.2 \\
Rara vez & 9 & 27.3 & 27.3 \\
Algunas veces & 7 & 21.2 & 21.2 \\
Casi siempre & 9 & 27.3 & 27.3 \\
Siempre & 2 & 6.1 & 6.1 \\
Total & 33 & 100.0 & 100.0 \\
\hline
\end{tabular}

En este ítem se pretende conocer la frecuencia con la que los estudiantes presentan sentimientos de tristeza cuando tienen actividades académicas, como resultado se obtuvo que el $21.2 \%$ nunca presentan estos sentimientos, el 30.3\% rara vez, el 33.3\% casi siempre, el $9.1 \%$ casi siempre y el $6.1 \%$ siempre, ver tabla 21.

Tabla 21

Sentimientos de tristeza

\begin{tabular}{llll}
\hline Sentimientos & Frecuencia & Porcentaje & Porcentaje válido \\
\hline Nunca & 7 & 21.2 & 21.2 \\
Rara vez & 10 & 30.3 & 30.3 \\
Algunas veces & 11 & 33.3 & 33.3 \\
Casi siempre & 3 & 9.1 & 9.1 \\
Siempre & 2 & 6.1 & 6.1 \\
Total & 33 & 100.0 & 100.0
\end{tabular}

Con este ítem se determinará la frecuencia con la que los jóvenes universitarios presentan angustia o desesperación con las labores académicas, el 24.2\% considera que nunca ha presentado angustia o desesperación, el $27.3 \%$ rara vez, el 21.2\% algunas veces al igual que casi siempre y únicamente el $6.1 \%$ considera que siempre (ver tabla 22).

Tabla 22

Angustia o desesperación

\begin{tabular}{llll}
\hline Angustia o desesperación & Frecuencia & Porcentaje & Porcentaje válido \\
\hline Nunca & 8 & 24.2 & 24.2 \\
Rara vez & 9 & 27.3 & 27.3 \\
Algunas veces & 7 & 21.2 & 21.2 \\
Casi siempre & 7 & 21.2 & 21.2
\end{tabular}




$\begin{array}{llll}\text { Siempre } & 2 & 6.1 & 6.1 \\ \text { Total } & 33 & 100.0 & 100.0\end{array}$

Este ítem tiene la finalidad de conocer la frecuencia con la que los estudiantes de psicología presentan problemas de concentración en la jornada escolar, el $24.2 \%$ determino que nunca al igual que algunas veces y casi siempre, el 18.2\% rara vez, y solo el $9.1 \%$ siempre (ver tabla 23).

Tabla 23

Problemas de concentración

\begin{tabular}{llll}
\hline Problemas & Frecuencia & Porcentaje & Porcentaje válido \\
\hline Nunca & 8 & 24.2 & 24.2 \\
Rara vez & 6 & 18.2 & 18.2 \\
Algunas veces & 8 & 24.2 & 24.2 \\
Casi siempre & 8 & 24.2 & 24.2 \\
Siempre & 3 & 9.1 & 9.1 \\
Total & 33 & 100.0 & 100.0 \\
\hline
\end{tabular}

Para conocer la frecuencia con la que los universitarios presentan sentimientos de agresividad o aumento de irritabilidad, se aplicó este ítem, en el cual se generaron los siguientes resultados: el $24.2 \%$ nunca presentan estos sentimientos, el 36.4\% rara vez, el $18.2 \%$ algunas veces, el $12.1 \%$ casi siempre y el $9.1 \%$ siempre (ver tabla 24 ).

Tabla 24

Sentimiento de agresividad o aumento de irritabilidad

\begin{tabular}{llll}
\hline Sentimiento & Frecuencia & Porcentaje & Porcentaje válido \\
\hline Nunca & 8 & 24.2 & 24.2 \\
Rara vez & 12 & 36.4 & 36.4 \\
Algunas veces & 6 & 18.2 & 18.2 \\
Casi siempre & 4 & 12.1 & 12.1 \\
Siempre & 3 & 9.1 & 9.1 \\
Total & 33 & 100.0 & 100.0 \\
\hline
\end{tabular}

Por último, se quiere conocer las reacciones comportamentales de los estudiantes y para ello se aplicaron cuatro ítems. El primer ítem se aplicó con la finalidad de conocer la frecuencia con la que los alumnos presentan conflicto o tienden a discutir cuando tienen labores escolares, como resultado se obtuvo que el $30.3 \%$ de los alumnos nunca y rara vez 
han presentado conflicto o han tendido a discutir, el $18.2 \%$ algunas veces, el $12.1 \%$ casi siempre y el $9.1 \%$ siempre (ver tabla 25 ).

Tabla 25

Conflicto o tendencia a discutir

\begin{tabular}{llll}
\hline Conflicto & Frecuencia & Porcentaje & Porcentaje válido \\
\hline Nunca & 10 & 30.3 & 30.3 \\
Rara vez & 10 & 30.3 & 30.3 \\
Algunas veces & 6 & 18.2 & 18.2 \\
Casi siempre & 4 & 12.1 & 12.1 \\
Siempre & 3 & 9.1 & 9.1 \\
Total & 33 & 100.0 & 100.0
\end{tabular}

Para conocer la frecuencia con la que los estudiantes presentan aislamiento de los demás se aplicó este ítem, en el cual los alumnos respondieron lo siguiente: el 39.4\% nunca, el 36.4\% rara vez, el 18.2\% algunas veces y solamente el $6.1 \%$ respondió casi siempre (ver tabla 26).

Tabla 26

Aislamiento de los demás

\begin{tabular}{llll}
\hline Aislamiento & Frecuencia & Porcentaje & Porcentaje válido \\
\hline Nunca & 13 & 39.4 & 39.4 \\
Rara vez & 12 & 36.4 & 36.4 \\
Algunas veces & 6 & 18.2 & 18.2 \\
Casi siempre & 2 & 6.1 & 6.1 \\
Total & 33 & 100.0 & 100.0 \\
\hline
\end{tabular}

Este ítem se implementó para determinar la frecuencia con la que los estudiantes presentan desgano para realizar las labores universitarias. El 24.2\% respondió que nunca, el $42.4 \%$ rara vez, el $18.2 \%$ algunas veces y el $15.2 \%$ casi siempre (ver tabla 27 ).

Tabla 27

Desgano para realizar las labores universitarias

\begin{tabular}{llll}
\hline Desgano & Frecuencia & Porcentaje & Porcentaje válido \\
\hline Nunca & 8 & 24.2 & 24.2 \\
Rara vez & 14 & 42.4 & 42.4 \\
Algunas veces & 6 & 18.2 & 18.2 \\
\hline
\end{tabular}


Casi siempre

Total
5

33
15.2

100.0
15.2

100.0

En el último ítem se desea conocer la frecuencia con la que los universitarios presentan aumento o reducción de consumo de alimentos, el 21.2\% respondió que nuca al igual que rara vez y casi siempre, el $27.3 \%$ algunas veces y solo el $9.1 \%$ siempre (ver tabla 28).

Tabla 28

Aumento o reducción del consumo de alimentos

\begin{tabular}{llll}
\hline Consumo de alimentos & Frecuencia & Porcentaje & Porcentaje válido \\
\hline Nunca & 7 & 21.2 & 21.2 \\
Rara vez & 7 & 21.2 & 21.2 \\
Algunas veces & 9 & 27.3 & 27.3 \\
Casi siempre & 7 & 21.2 & 21.2 \\
Siempre & 3 & 9.1 & 9.1 \\
Total & 33 & 100.0 & 100.0 \\
\hline
\end{tabular}

\section{Conclusiones y Recomendaciones}

En este apartado se presentan las conclusiones y recomendaciones a las cuales se llegaron al terminar con la investigación sobre los efectos del estrés en el rendimiento académico de los estudiantes de la carrera de psicología, llevada a cabo en el Instituto Tecnológico de Sonora, campus Navojoa.

\section{Conclusiones:}

Se llegó a la conclusión que existen muchas causas que generan estrés y esto influye en el rendimiento académico de los jóvenes universitarios. Algunas de ellas son la carga excesiva de tareas y trabajos con un alto nivel de exigencia por parte de los docentes, el tiempo limitado para realizar dichas actividades y en algunas ocasiones la competencia que existe entre compañeros de grupo. Otra de las causas que sobresale en la influencia del estrés, es la implementación de las evaluaciones de los docentes como son los exámenes, trabajos finales o trabajos de investigación.

$\mathrm{Al}$ igual de las causas existen algunos factores que influyen en la generación de estrés en los estudiantes, como es la falta de motivación por parte de los docentes y esto afecta en sus labores académicas y en lo personal. La motivación es fundamental para que 
los alumnos se sientan con las capacidades de poder realizar cualquier actividad escolar que sea asignada por los docentes, sin importar que tenga un nivel alto de dificultad para realizarla.

Algunos de los efectos físicos que pueden ocasionar estrés en los estudiantes de psicología son: los trastornos de sueño como es el insomnio y las pesadillas, al igual que la presencia de fatiga crónica que tiene como padecimiento siempre sentirse cansado, así como la aparición de dolores de cabeza o migraña y la ansiedad de morderse las uñas. Asimismo, existen efectos psicológicos que originan el estrés en los alumnos como son: los sentimientos de tristeza, la incapacidad de relajarse, los problemas de concentración para realizar cualquier labor, la angustia con desesperación y la falta de apetito.

Todos estos efectos tanto físicos como psicológicos son generados por diversas causas o factores, dependiendo de las circunstancias, acciones o acontecimientos de cada uno de los estudiantes. El entorno social también influye, al igual que la relación con sus familiares en casa o con sus amigos, pareja, entre otros.

\section{Recomendaciones:}

1. Los Maestros de todas las instituciones deben de crear un buen ambiente de aprendizaje con una buena motivación, para el que los alumnos desarrollen su máximo potencial.

2. Otra recomendación importante es que los estudiantes duerman por lo menos 8 horas al día, para que no afecte en su rendimiento académico y para que no se provoquen estrés.

3. Sugerirle al alumnado practicar relajación y respiración profunda cuando se sientan fatigados u estresados.

4. El estudiante debe esforzarse en mantener la calma y tranquilidad cuando se encuentre elaborando tareas que se le dificulten.

5. Organización de actividades por parte de los alumnos para su elaboración, para evitar la acumulación de tareas o la carga excesiva de trabajos.

Las recomendaciones anteriores pueden ayudar a combatir el estrés, disminuyendo su nivel. Cabe mencionar que según la investigación de Tolentino (2009) el estrés alcanza una mayor problemática en los estudios de nivel superior, ya que se caracterizan por las altas cargas académicas que se presentan en la universidad, es por ello que se recomienda a 
los estudiantes la organización de sus actividades para evitar la acumulación de tareas, y así no caer en la problemática que es el estrés académico.

Además, la investigación de Kadapatti y Vijayalaxmi (2012) citado por Domínguez, Guerrero y Castillo (2015), mencionan que no solo la carga académica afecta a los estudiantes universitarios, sino también las relaciones familiares, así como las sociales. Para ello la mejor solución a ello es practicar relajación y respiración profunda cuando se sientan estresados por esos factores.

\section{Referencias}

Alvarado, L. (2015, agosto). "Motivación al logro de los estudiantes de primero y noveno ciclo de administración de empresas de las sedes de la universidad Rafael landívar en jutiapa y escuintla". http://recursosbiblio.url.edu.gt/tesiseortiz/2015/05/83/AlvaradoLuisana.pdf

Barraza, A. (2007, 1 julio). El campo de estudio del estrés: del Programa de Investigación Estímulo-Respuesta al Programa de Investigación Persona-Entorno. Revista internacional de psicología. http://www.revistapsicologia.org/index.php/revista/article/view/48

Barraza, A. (2010, 6 octubre). Propiedades psicométricas del Inventario SISCO del estrés académico. Revista Psicología Científica http://www.psicologiacientifica.com/siscopropiedades-psicometricas/

Berrío, N., y Mazo, R. (2011). Estrés Académico. Revista de Psicología Universidad de Antioquia.

https://aprendeenlinea.udea.edu.co/revistas/index.php/psicologia/article/view/11369/1 0646

Caldera, J., Pulido, B., y Martínez, M. (2007). Niveles de estrés y rendimiento académico en estudiantes de la carrera de Psicología del Centro Universitario de Los Altos. Revista Educación y Desarrollo. http://www.cucs.udg.mx/revistas/edu_desarrollo/anteriores/7/007_Caldera.pdf Campo, F. (2017). La búsqueda de sentido como factor motivador del estudiante en clase de religión. 
http://repositorio.uft.cl/xmlui/bitstream/handle/20.500.12254/1323/DEL\%20CAMPO \%20FRANCISCO \%202017.pdf? sequence=1\&isAllowed=y

Domínguez, R., Guerrero, G., y Castillo, J. (2015). Influencia del estrés en el rendimiento académico de un grupo de estudiantes universitarios.

http://www.educacionyciencia.org/index.php/educacionyciencia/article/download/313 /pdf_12

Encina, Y., y Ávila, M. (2015). Validación de una escala de estrés cotidiano en escolares chilenos. Revista de Psicología. https://www.redalyc.org/pdf/3378/337839590005.pdf

Erazo, O. (2012, 15 febrero). El rendimiento académico, un fenómeno de múltiples relaciones y complejidades. Revista Vanguardia Psicológica. https://dialnet.unirioja.es/descarga/articulo/4815141.pdf

Fernández, J. y Luévano, E. (2018). Influencia del estrés académico sobre el rendimiento escolar en educación media superior. Revista Panamericana de pedagogía dehttp://portalderevistasdelaup.mx/revistapedagogia/index.php/pedagogia/article/dow $\operatorname{nload} / 284 / 287$

Fernández, R. (2017, 21 marzo). Aproximación del hecho científico en educación infantil. Revista Digital de futuros maestros en la Facultad de Educación de Toledo. https://revistamagisterioelrecreo.blogspot.com/2019/10/aproximacion-del-hechocientifico-en.html

García, A., y Basilotta, V. (2017, 9 febrero). Aprendizaje Basado en Proyectos (ABP): evaluación desde la perspectiva de alumnos de Educación Primaria. Revista de Investigación Educativa. https://revistas.um.es/rie/article/view/246811/203561

García, R., Pérez, F., y Natividad, L. (2011, noviembre). Evaluación del estrés académico en estudiantes de nueva incorporación a la universidad. Revista latinoamericana de psicología. https://www.redalyc.org/pdf/805/80524058011.pdf

González, C., Niebla, J., Díaz, K., y López, M. (2012, 17 marzo). Rendimiento académico y factores asociados. Aportaciones de algunas evaluaciones a gran escala. https://dialnet.unirioja.es/descarga/articulo/3960787.pdf 
González, R., Valle, A., Nuñez, J., y González, J. (2014). Una aproximación teórica al concepto de metas académicas y su relación con la motivación escolar. http://www.psicothema.com/psicothema.asp?id=4

Gómez, H. y Sánchez, V. (2013). Indicadores cualitativos para la medición de la calidad en la educación. Educ. Educ. Vol. 16, No. 1, pp. 9-24.

Hernández, R., Fernández, C., y Baptista, P. (2014). Metodología de la investigación (6 ed). Distrito Federal, México: McGraw-Hill.

Instituto Tecnológico de Sonora, (2015) Plan de Desarrollo Institucional 2020. Instituto Tecnológico de Sonora. https://www.itson.mx/universidad/Paginas/dpi.aspx

Instituto Tecnológico de Sonora, (2019) Sistema integral de trayectorias escolares. Instituto Tecnológico de Sonora.

Kadapatti, M. y Vijayalaxmi, A. (2012). Stressors of academic stress. https://www.ijsr.in/upload/428127980Paper\%20\%2030.pdf

Lamas, H. (2015, 30 abril). Sobre el rendimiento escolar. Propósitos y representaciones. https://dialnet.unirioja.es/servlet/articulo?codigo=5475216

Molina, M. (2015, diciembre). Valoración de los criterios referentes al rendimiento académico y variables que lo puedan afectar. Revista Médica Electrónica. http://scielo.sld.cu/scielo.php?script=sci_arttext\&pid=S1684-18242015000600007

Pozos, B., Preciado, M., Plascencia, A., Acosta, M., y Aguilera, M. (2015). Estrés académico y síntomas físicos, psicológicos y comportamentales en estudiantes mexicanos de una universidad pública. https://dialnet.unirioja.es/servlet/articulo?codigo $=6850073$

Pulido, F., y Herrera, F. (2017). La influencia de las emociones sobre el rendimiento académico. https://www.redalyc.org/jatsRepo/4595/459551482004/459551482004.pdf

Pulido, M., Serrano, M., Valdés, E., Chávez, M., Hidalgo, P., y Vera, F. (2011). Estrés académico en estudiantes universitarios. Instituto de Investigaciones Psicológicas de la Universidad Veracruzana.

http://psicologiaysalud.uv.mx/index.php/psicysalud/article/view/584 
Rivera, G. (2014). La motivación del alumno y su relación con el rendimiento académico en los estudiantes de Bachillerato Técnico en Salud Comunitaria del Instituto República. http://www.cervantesvirtual.com/obra/la-motivacion-del-alumno-y-surelacion-con-el-rendimiento-academico-en-los-estudiantes-de-bachillerato-tecnicoen-salud-comunitaria-del-instituto-republica-federal-de-mexico-de-comayaguelamdc-durante-el-ano-lectivo-2013/

Román, C. y Hernandéz, H. (2011). El estrés académico: una revisión crítica del concepto desde las ciencias de la educación. Revista Electrónica de Psicología Iztacala. http://www.revistas.unam.mx/index.php/repi/article/view/26023

Sánchez, A., y Pérez, P. (2017, 12 julio). La formación humanista. Un encargo para la educación. Universidad y Sociedad. http://rus.ucf.edu.cu/index.php/rus

Sánchez, D., Oviedo, R., y Martínez, E. (2011, 26 junio). Factores que influyen en el rendimiento académico del estudiante universitario. http://tecnociencia.uach.mx/numeros/v5n2/data/Factores_que_influyen_en_el_rendim iento_academico_del_estudiante_universitario.pdf

Suárez, N., y Díaz, L. (2014, 17 diciembre). Estrés académico, deserción y estrategias de retención de estudiantes en la educación superior. http://www.scielo.org.co/pdf/rsap/v17n2/v17n2a13.pdf

Tolentino, S. (2009, febrero). Perfil de estrés académico en alumnos de licenciatura en Psicología, de la Universidad Autónoma de Hidalgo en la Escuela Superior de Actopan.

http://dgsa.uaeh.edu.mx:8080/bibliotecadigital/bitstream/handle/231104/243/Perfil\% $20 \mathrm{de} \% 20 \mathrm{estres} \% 20$ academico\%20en $\% 20$ alumnos.pdf?sequence=1\&isAllowed=y

Cómo citar:

Barrón Miranda, M., \& Armenta Zazueta, L. . (2021). Efectos del estrés en el rendimiento académico de los Estudiantes de la Licenciatura de Psicología. Revista De Investigación Académica Sin Frontera: División De Ciencias Económicas Y Sociales, (36). https://doi.org/10.46589/rdiasf.vi36.388 


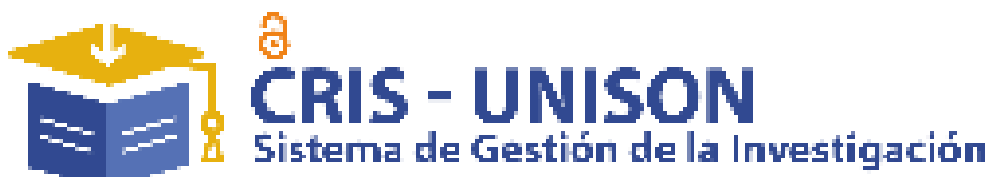

- Dial net

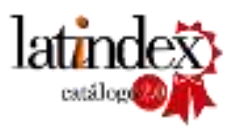

If IIIFACTOR

REDIB

RED

Citeractor

2LatinREV $V_{\text {Neliti - Indonesia's Research Repository }}$

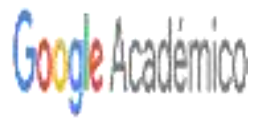

DORA

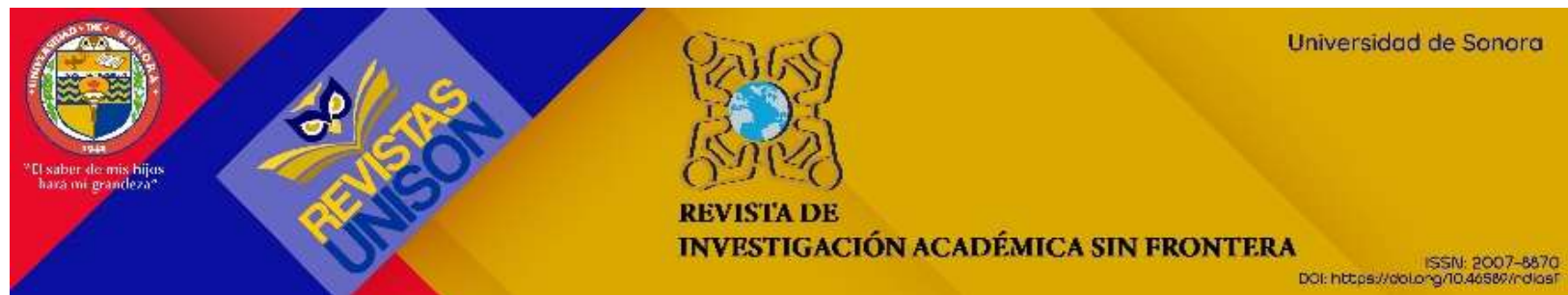

